\title{
Hopf Bifurcations and Oscillatory Patterns of a Homogeneous Reaction-Diffusion Singular Predator-Prey Model
}

\author{
Zhenhua Bao ${ }^{1,2}$ and He Liu ${ }^{3}$ \\ ${ }^{1}$ School of Mathematical Sciences, Dalian University of Technology, Dalian 116024, China \\ ${ }^{2}$ School of Mathematics, Liaoning Normal University, Dalian 116029, China \\ ${ }^{3}$ School of Mathematics, Physics and Biological Engineering, Inner Mongolia University of Science and Technology, \\ Baotou 014010, China \\ Correspondence should be addressed to He Liu; younglust@163.com
}

Received 7 March 2013; Accepted 23 August 2013

Academic Editor: L. Jódar

Copyright @ 2013 Z. Bao and H. Liu. This is an open access article distributed under the Creative Commons Attribution License, which permits unrestricted use, distribution, and reproduction in any medium, provided the original work is properly cited.

\begin{abstract}
A kind of homogeneous reaction-diffusion singular predator-prey model with no-flux boundary condition is considered. By using the abstract simplified Hopf bifurcation theorem due to Yi et al. 2009, we performed detailed Hopf bifurcation analysis of this particular pattern formation system. These results suggest the existence of oscillatory patterns if the system parameters fall into certain parameter ranges. And all these oscillatory patterns are proved to be unstable.
\end{abstract}

\section{Introduction}

In this paper, we consider the following reaction-diffusion singular predator-prey model:

$$
\begin{gathered}
u_{t}=d_{1} u_{x x}+\alpha(1-u) u-v, \quad x \in(0, \ell \pi), t>0, \\
v_{t}=d_{2} v_{x x}+\beta v\left(1-\frac{v}{u}\right), \quad x \in(0, \ell \pi), t>0, \\
u(x, 0)=u_{0}(x), \quad v(x, 0)=v_{0}(x), \quad x \in(0, \ell \pi), \\
u_{x}^{\prime}(x, t)=v_{x}^{\prime}(x, t)=0, \quad x=0, \ell \pi, t>0,
\end{gathered}
$$

where $u(x, t)$ and $v(x, t)$ are the population densities of the prey and predator at time $t$ and position $x, \alpha$ and $\beta$ are dimensionless positive parameters; $\ell \in(0, \infty)$. The underlying spatially homogeneous problem was derived in [1] to model prey-predator interactions in fragile (insular) environments. The spatially structured system (1) supplemented with initial data and no-flux boundary conditions was introduced in [2]. For more information on the system (1), we refer the reader to $[1,2]$ for great details.

Mathematically, the model was considered by Ducrot and Langlais [3], where the authors first provided a suitable notion of global travelling wave weak solution. They mainly studied the existence of travelling waves solutions for predator invasion in such environments. Under suitable conditions on the diffusion coefficients and on species growth rates they were able to prove that the travelling wave solutions were actually positive on a half line and identically zero elsewhere.

The present paper is targeting to consider the Hopf bifurcations of the reaction-diffusion system (1), by using the simplified Hopf bifurcation theorem due to [4], which is widely used to prove the existence of oscillatory patterns of different kind of pattern formation systems, including GiererMinhardt model [5], Degn-Harrison model [6], bimolecular model [7], hair growth controlling model [8], and the Sel'kov model [9].

Our results show that, under suitable choice of system parameters, system (1) will undergo spatially homogeneous and nonhomogeneous oscillatory phenomena. And once the oscillatory patterns exist, they are always unstable. These unstable patterns cannot be observed by numerical simulations; thus, numerical simulations corresponding to our analytical analysis are unavailable in the paper, even though, the analytical results we obtained allow for the clearer understanding of the rich dynamics of the system.

The rest of this paper is structured in the following way. In Section 2, we perform Hopf bifurcation analysis of the 
system. In Section 3, we draw some concluding remarks to end up our discussion. Throughout the paper, we denote by $\mathbb{N}$ the set of all the positive integers and $\mathbb{N}_{0}=\mathbb{N} \cup\{0\}$.

\section{Stability and Hopf Bifurcation Analysis}

To begin with, for convenience of our discussion, we copy (1) in the following:

$$
\begin{gathered}
u_{t}=d_{1} u_{x x}+\alpha(1-u) u-v, \quad x \in(0, \ell \pi), t>0, \\
v_{t}=d_{2} v_{x x}+\beta v\left(1-\frac{v}{u}\right), \quad x \in(0, \ell \pi), t>0, \\
u(x, 0)=u_{0}(x), \quad v(x, 0)=v_{0}(x), \quad x \in(0, \ell \pi), \\
u_{x}^{\prime}(x, t)=v_{x}^{\prime}(x, t)=0, \quad x=0, \ell \pi, t>0 .
\end{gathered}
$$
with

System (2) has a unique equilibrium solution $\left(u_{\alpha}, v_{\alpha}\right)$,

$$
u_{\alpha}=v_{\alpha}=: \frac{\alpha-1}{\alpha},
$$

which is in the first quadrant if and only if $\alpha>1$.

In the following, we always assume that $\alpha>1$ holds. We fix the parameter $\beta$ and choose $\alpha$ as the bifurcation parameter.

The linearized operator of system (2) evaluated at $\left(u_{\alpha}, v_{\alpha}\right)$ is given by

$$
L(\alpha):=\left(\begin{array}{cc}
d_{1} \frac{\partial^{2}}{\partial x^{2}}+(2-\alpha) & -1 \\
\beta & d_{2} \frac{\partial^{2}}{\partial x^{2}}-\beta
\end{array}\right) .
$$

It follows from $[4,10]$ that the eigenvalues of $L(\alpha)$ are given by these of the operator $L_{n}(\alpha)$, which is defined by

$$
L_{n}(\alpha):=\left(\begin{array}{cc}
-\frac{d_{1} n^{2}}{\ell^{2}}+(2-\alpha) & -1 \\
\beta & -\frac{d_{2} n^{2}}{\ell^{2}}-\beta
\end{array}\right),
$$

whose characteristic equation is

$$
\lambda^{2}-\lambda T_{n}(\alpha)+D_{n}(\alpha)=0, \quad n=0,1,2, \ldots,
$$

where

$$
\begin{aligned}
T_{n}(\alpha)=(2-\alpha & -\beta)-\frac{\left(d_{1}+d_{2}\right) n^{2}}{\ell^{2}}:=A(\alpha)-\frac{\left(d_{1}+d_{2}\right) n^{2}}{\ell^{2}}, \\
D_{n}(\alpha)= & \frac{d_{1} d_{2} n^{4}}{\ell^{4}} \\
& +\frac{n^{2}}{\ell^{2}}\left[d_{1} \beta-d_{2}(2-\alpha)\right]+\beta(\alpha-1) .
\end{aligned}
$$

We have the following theorem on the stability of the unique positive equilibrium solution $\left(u_{\alpha}, v_{\alpha}\right)$.
Theorem 1. Suppose that one of the following conditions holds

(1) $\beta \geq 1$ and $\alpha>1$,

(2) $0<\beta<1$ and $\alpha>2-\beta$

and that $d_{1}, d_{2}>0$ such that

$$
\frac{d_{1}}{d_{2}} \geq \frac{1}{\beta}
$$

is satisfied. Then, $\left(u_{\alpha}, v_{\alpha}\right)$ is always locally asymptotically stable in the reaction-diffusion equation (2).

Proof. On one hand, for any $\alpha>1$, we have

$$
D_{n}(\alpha)>\frac{d_{1} d_{2} n^{4}}{\ell^{4}}+\frac{n^{2}}{\ell^{2}}\left(d_{1} \beta-d_{2}\right),
$$

which implies that $D_{n}(\alpha)>0$ always holds for any $\alpha \in(1, \infty)$ if

$$
\frac{d_{1}}{d_{2}} \geq \frac{1}{\beta}
$$

holds.

On the other hand, if $\beta \geq 1$ and $\alpha>1$, or $0<\beta<1$ but $\alpha>2-\beta$ holds, then we can obtain that, for any $n \in$ $\mathbb{N}_{0}$ and $\alpha>0$, we always have $T_{n}(\alpha)<0$. Thus, $\left(u_{\alpha}, v_{\alpha}\right)$ is always locally asymptotically stable in the reaction-diffusion equation (2).

Now we consider the Hopf bifurcations of the system. According to [4], a point $\alpha^{H} \in(1, \infty)$ is a Hopf bifurcation point if and only if there exists $n \in \mathbb{N}_{0}$, such that

$$
\begin{gathered}
T_{n}\left(\alpha^{H}\right)=0, \quad D_{n}\left(\alpha^{H}\right)>0 ; \\
T_{j}\left(\alpha^{H}\right) \neq 0, \quad D_{j}\left(\alpha^{H}\right) \neq 0 \quad \text { for } j \neq n ;
\end{gathered}
$$

and $\tau^{\prime}\left(\alpha^{H}\right) \neq 0$, where $\tau(\alpha)$ is the real parts of the unique pair of complex eigenvalues $\tau(\alpha) \pm i \omega(\alpha)$ near the imaginary axis.

Thus, any potential Hopf bifurcation point $\alpha^{H}$ must be in the interval $(1,2-\beta]$, where we assume that $0<\beta<1$.

For any Hopf bifurcation point $\alpha^{H} \in(1,2-\beta]$, let $\tau(\alpha) \pm$ $i \omega(\alpha)$ be the eigenvalues of $L_{n}(\alpha)$ near $\alpha=\alpha^{H}$ then we have

$$
\begin{gathered}
\tau(\alpha)=\frac{A(\alpha)}{2}-\frac{\left(d_{1}+d_{2}\right) n^{2}}{2 \ell^{2}}, \\
\omega(\alpha)=\sqrt{D_{n}(\alpha)-\tau^{2}(\alpha) .}
\end{gathered}
$$

Then, for any $\alpha \in(1,2-\beta]$, we have

$$
\tau^{\prime}(\alpha)=-\frac{1}{2} .
$$

This implies that the transversality condition $\tau^{\prime}(\alpha) \neq 0$ is always satisfied for any $\alpha \in(1,2-\beta]$.

Suppose that $0<\beta<1$ holds and define

$$
\ell_{n}=\frac{n}{\sqrt{(1-\beta) /\left(d_{1}+d_{2}\right)}}, \quad n \in \mathbb{N}_{0} .
$$


Then, for any $\ell_{n}<\ell \leq \ell_{n+1}$ and $1 \leq j \leq n$, we define $\alpha_{j}^{H}$ as the roots of

$$
A(\alpha)=\frac{\left(d_{1}+d_{2}\right) j^{2}}{\ell^{2}}
$$

satisfying $1<\alpha_{j}^{H}<\alpha_{0}^{H}:=2-\beta$, and these points satisfy

$$
1<\alpha_{n}^{H}<\alpha_{n-1}^{H}<\cdots<\alpha_{1}^{H}<\alpha_{0}^{H}=2-\beta
$$

and $\lim _{j \rightarrow \infty} \alpha_{j}^{H}=1$. Clearly $T_{j}\left(\alpha_{j}^{H}\right)=0$ and $T_{i}\left(\alpha_{j}^{H}\right) \neq 0$ for $i \neq j$.

Now we derive a condition from the parameters so that $D_{n}(\alpha)>0$ for all $\alpha \in(1,2-\beta]$ and $n \in \mathbb{N}_{0}$. In fact, from (8) in Theorem 1, it follows that $D_{n}(\alpha)>0$ always holds.

Based on the discussions above, we have the following Hopf bifurcation results for the reaction-diffusion model (2).

Theorem 2. Suppose that the constants $d_{1}, d_{2}, \beta$ satisfying the condition (8) and $\ell_{n}$ are defined as in (14). Then, for any $\ell$ in $\left(\ell_{n}, \ell_{n+1}\right]$, there exist $n$ points $\alpha_{j}^{H}(\ell), 1 \leq j \leq n$, satisfying

$$
1<\alpha_{n}^{H}<\alpha_{n-1}^{H}<\cdots<\alpha_{1}^{H}<\alpha_{0}^{H}=2-\beta,
$$

such that the system (1) undergoes a Hopf bifurcation at $\alpha=\alpha_{j}^{H}$ or $\alpha=\alpha_{0}^{H}$. Moreover,

(1) the bifurcating periodic solutions from $\alpha=\alpha_{0}^{H}$ are spatially homogeneous, which coincides with the periodic solution of the corresponding ODE system;

(2) the bifurcating periodic solutions from $\alpha=\alpha_{j}^{H}$ are spatially nonhomogeneous.

Next we consider the bifurcation direction and stability of the bifurcating periodic solutions.

Theorem 3. For the system (2), the direction of the Hopf bifurcation at $\alpha=\alpha_{0}^{H}$ is forward, and the bifurcating (spatial homogeneous) periodic solutions are unstable.

Proof. By Theorem 2.1 of [4], in order to determine the stability and bifurcation direction of the bifurcating periodic solution, we need to calculate $\operatorname{Re}\left(c_{1}\left(\alpha_{0}^{H}\right)\right)$. When $\alpha=\alpha_{0}^{H}$, we put

$$
\begin{gathered}
q=\left(\begin{array}{l}
a_{0} \\
b_{0}
\end{array}\right)=\left(\begin{array}{c}
1 \\
\beta-\omega_{0} i
\end{array}\right), \\
q^{*}=\left(\begin{array}{l}
a_{0}^{*} \\
b_{0}^{*}
\end{array}\right)=\left(\begin{array}{c}
\frac{\omega_{0}+\beta i}{2 \omega_{0} \ell \pi} \\
-\frac{i}{2 \omega_{0} \ell \pi}
\end{array}\right),
\end{gathered}
$$

where $\omega_{0}=\sqrt{\beta(1-\beta)}$.
Now translate (2) into the following system by the translation $\widehat{u}=u-u_{\alpha}$ and $\widehat{v}=v-v_{\alpha}$, and still let $u$ and $v$ denote $\widehat{u}$ and $\widehat{v}$ for the convenience of notation. We have

$$
\begin{gathered}
u_{t}-d_{1} u_{x x}=\alpha\left[1-\left(u+u_{\alpha}\right)\right]\left(u+u_{\alpha}\right)-\left(v+v_{\alpha}\right), \\
x \in(0, \ell \pi), \quad t>0, \\
v_{t}-d_{2} v_{x x}=\beta\left(v+v_{\alpha}\right)\left(1-\frac{v+v_{\alpha}}{u+u_{\alpha}}\right), \\
x \in(0, \ell \pi), \quad t>0, \\
u(x, 0)=u_{0}(x), \quad v(x, 0)=v_{0}(x), \quad x \in(0, \ell \pi), \\
u_{x}^{\prime}(x, t)=v_{x}^{\prime}(x, t)=0, \quad x=0, \ell \pi, \quad t>0 .
\end{gathered}
$$

Following [4], we define

$$
\begin{gathered}
f(\alpha, u, v)=\alpha\left[1-\left(u+u_{\alpha}\right)\right]\left(u+u_{\alpha}\right)-\left(v+v_{\alpha}\right), \\
g(\alpha, u, v)=\beta\left(v+v_{\alpha}\right)\left(1-\frac{v+v_{\alpha}}{u+u_{\alpha}}\right) .
\end{gathered}
$$

By (2.19) of [4], we have

$$
\begin{gathered}
c_{0}=e_{0}=2(\beta-2), \\
d_{0}=\frac{2 \beta(\beta-2)}{1-\beta}\left(1-\beta+\omega_{0} i\right)^{2}, \\
f_{0}=\beta(\beta-2), \quad g_{0}=0, \\
h_{0}=\frac{2 \beta(2-\beta)^{2}}{1-\beta}\left(3-2 \beta+2 \omega_{0} i\right) .
\end{gathered}
$$

Following [4], we define

$$
Q_{q q}=\left(\begin{array}{c}
c_{0} \\
d_{0}
\end{array}\right), \quad Q_{q \bar{q}}=\left(\begin{array}{c}
e_{0} \\
f_{0}
\end{array}\right), \quad C_{q q \bar{q}}=\left(\begin{array}{c}
g_{0} \\
f_{0}
\end{array}\right) .
$$

Note that $\langle\cdot, \cdot\rangle$ is the complex-value inner product defined as

$$
\left\langle U_{1}, U_{2}\right\rangle=\int_{0}^{\ell \pi}\left(\bar{u}_{1} u_{2}+\bar{v}_{1} v_{2}\right) d x
$$

with $U_{i}=\left(u_{i}, v_{i}\right)^{T}, i=1,2$.

Then we obtain easily that

$$
\begin{gathered}
\left\langle q^{*}, Q_{q q}\right\rangle=\frac{\beta-2}{\omega_{0}}\left[(1-2 \beta) \omega_{0}-2 \beta^{2} i\right], \\
\left\langle\bar{q}^{*}, Q_{q q}\right\rangle=\frac{\beta-2}{\omega_{0}}\left[(1+2 \beta) \omega_{0}+2 \beta^{2} i\right], \\
\left\langle q^{*}, Q_{q \bar{q}}\right\rangle=\left\langle\bar{q}^{*}, Q_{q \bar{q}}\right\rangle=\beta-2, \\
\left\langle q^{*}, C_{q q \bar{q}}\right\rangle=\frac{\beta(2-\beta)^{2}}{\omega_{0}(1-\beta)}\left[-2 \omega_{0}+(3-2 \beta)\right] .
\end{gathered}
$$


Hence it is straightforward to calculate that

$$
\begin{aligned}
& H_{20}=\left(\begin{array}{c}
c_{0} \\
d_{0}
\end{array}\right)-\left\langle q^{*}, Q_{q q}\right\rangle\left(\begin{array}{l}
a_{0} \\
b_{0}
\end{array}\right)-\left\langle\bar{q}^{*}, Q_{q q}\right\rangle\left(\frac{\overline{a_{0}}}{b_{0}}\right)=\left(\begin{array}{l}
0 \\
0
\end{array}\right), \\
& H_{11}=\left(\begin{array}{l}
e_{0} \\
f_{0}
\end{array}\right)-\left\langle q^{*}, Q_{q \bar{q}}\right\rangle\left(\begin{array}{l}
a_{0} \\
b_{0}
\end{array}\right)-\left\langle\bar{q}^{*}, Q_{q \bar{q}}\right\rangle\left(\begin{array}{l}
\overline{a_{0}} \\
b_{0}
\end{array}\right)=\left(\begin{array}{l}
0 \\
0
\end{array}\right) .
\end{aligned}
$$

By (25), we have

$$
\begin{gathered}
w_{20}=\left[2 i \omega_{0} I-L\left(\alpha_{0}^{H}\right)\right]^{-1} H_{20}=\left(\begin{array}{l}
0 \\
0
\end{array}\right), \\
w_{11}=-\left[L\left(\alpha_{0}^{H}\right)\right]^{-1} H_{11}=\left(\begin{array}{l}
0 \\
0
\end{array}\right),
\end{gathered}
$$

where $I$ is the identity matrix. The equalities in (26) lead to

$$
\left\langle q^{*}, Q_{w_{11} q}\right\rangle=\left\langle q^{*}, Q_{w_{20} \bar{q}}\right\rangle=0 .
$$

Therefore, we can calculate that

$$
\begin{aligned}
& \operatorname{Re}\left(c_{1}\left(\alpha_{0}^{H}\right)\right) \\
& =\operatorname{Re}\left\{\frac{i}{2 \omega_{0}}\left\langle q^{*}, Q_{q q}\right\rangle \cdot\left\langle q^{*}, Q_{q \bar{q}}\right\rangle+\left\langle q^{*}, Q_{w_{11} q}\right\rangle\right. \\
& \left.+\frac{1}{2}\left\langle q^{*}, C_{q q \bar{q}}\right\rangle+\frac{1}{2}\left\langle q^{*}, Q_{w_{20} \bar{q}}\right\rangle\right\} \\
& =\operatorname{Re}\left\{\frac{(2-\beta)^{2}}{2 \omega_{0}^{2}}\left[2 \beta^{2}+(1-2 \beta) \omega_{0} i\right]\right. \\
& \left.+\frac{\beta(2-\beta)}{2 \omega_{0}(1-\beta)}\left[-2 \omega_{0}+(3-2 \beta) i\right]\right\} \\
& =\beta(2-\beta)>0 .
\end{aligned}
$$

From (13), it follows that $\tau^{\prime}\left(\alpha_{0}^{H}\right)=-1 / 2<0$, and then by Theorem 2.2 of [4], the direction of the Hopf bifurcation is forward and the bifurcating periodic solutions are unstable since $\operatorname{Re}\left(c_{1}\left(\alpha_{0}^{H}\right)\right)>0$.

Remark 4. (1) The direction of Hopf bifurcations at $\alpha=\alpha_{j}^{H}$ with $j \geq 1$ can also be calculated as what we did in Theorem 3, using the abstract results due to [4]. However, the calculations are very complicated. Thus, here we did not calculate the bifurcation direction of Hopf bifurcations at $\alpha=\alpha_{j}^{H}$ with $j \geq 1$.

(2) The spatial nonhomogeneous periodic solutions at $\alpha=\alpha_{j}^{H}$ with $j \geq 1$ found in Theorem 2 are clearly unstable since the steady state $\left(u_{\alpha}, v_{\alpha}\right)$ is unstable.

(3) Since all the periodic solutions are unstable, the simulations of the oscillatory patterns are unavailable here.

\section{Concluding Remarks}

In this paper, we considered a kind of diffusive GaucelLangleis model. By using the simplified Hopf bifurcation theory due to [4], we were capable of investigating the existence of Hopf bifurcations of the system, which indicates the existence of oscillatory patterns of the system. Our main bifurcation and stability analysis results in the paper can be summarized as follows.

(1) If $\beta$ is sufficiently large, say, if $\beta \geq 1$ holds, then Hopf bifurcations will never be possible. In fact, we can show that if one of the following conditions holds
(a) $\beta \geq 1$ and $\alpha>1$,
(b) $0<\beta<1$ and $\alpha>2-\beta$,

and $d_{1}, d_{2}>0$ such that $d_{1} / d_{2} \geq 1 / \beta$ is satisfied, then, $\left(u_{\alpha}, v_{\alpha}\right)$ is always locally asymptotically stable in the reaction-diffusion equation (2) (Theorem 1).

(2) However, if $\beta$ is not that large, say, $0<\beta<1$, then, Hopf bifurcation is possible in suitable parameter ranges. In fact, by choosing $\alpha$ as the bifurcation parameter, we can obtain that if the constants $d_{1}, d_{2}, \beta$ satisfying the condition (14) and $\ell_{n}$ are defined as in (14), then, for any $\ell$ in $\left(\ell_{n}, \ell_{n+1}\right]$, there exist $n$ points $\alpha_{j}^{H}(\ell), 1 \leq j \leq n$, satisfying

$$
1<\alpha_{n}^{H}<\alpha_{n-1}^{H}<\cdots<\alpha_{1}^{H}<\alpha_{0}^{H}=2-\beta,
$$

such that the system (1) undergoes a Hopf bifurcation at $\alpha=\alpha_{j}^{H}$ or $\alpha=\alpha_{0}^{H}$, (Theorem 2). Moreover,

(a) the bifurcation direction of Hop bifurcations at $\alpha=\alpha_{0}^{H}$ is forward and the bifurcating periodic solutions are unstable (Theorem 3 ),

(b) the bifurcation direction of Hopf bifurcations at $\alpha=\alpha_{j}^{H}$ with $j \geq 1$ can also be calculated as what we did in Theorem 3, using the abstract results due to [4]. However, the calculations are very complicated. This is beyond the scope of this paper,

(c) the spatial nonhomogeneous periodic solutions at $\alpha=\alpha_{j}^{H}$ with $j \geq 1$ found in Theorem 2 are clearly unstable since the steady state $\left(u_{\alpha}, v_{\alpha}\right)$ is unstable (Remark 4).

\section{Acknowledgment}

The authors are grateful to the anonymous referee for the comments and suggestions which definitely led to an improved presentation of the paper.

\section{References}

[1] F. Courchamp and G. Sugihara, "Modeling the biological control of an alien predator to protect island species from extinction," Ecological Applications, vol. 9, no. 1, pp. 112-123, 1999.

[2] S. Gaucel and M. Langlais, "Some remarks on a singular reaction-diffusion system arising in predator-prey modeling," Discrete and Continuous Dynamical Systems B, vol. 8, no. 1, pp. 61-72, 2007. 
[3] A. Ducrot and M. Langlais, "A singular reaction-diffusion system modelling prey-predator interactions: invasion and coextinction waves," Journal of Differential Equations, vol. 253, no. 2, pp. 502-532, 2012.

[4] F. Yi, J. Wei, and J. Shi, "Bifurcation and spatiotemporal patterns in a homogeneous diffusive predator-prey system," Journal of Differential Equations, vol. 246, no. 5, pp. 1944-1977, 2009.

[5] J. Liu, F. Yi, and J. Wei, "Multiple bifurcation analysis and spatiotemporal patterns in a 1-D gierermeinhardt model of morphogenesis," International Journal of Bifurcation and Chaos, vol. 20, no. 4, pp. 1007-1025, 2010.

[6] R. Peng, F. Yi, and X. Zhao, "Spatiotemporal patterns in a reaction-diffusion model with the Degn-Harrison reaction scheme," Journal of Differential Equations, vol. 254, no. 6, pp. 2465-2498, 2013.

[7] F. Yi, J. Liu, and J. Wei, "Spatiotemporal pattern formation and multiple bifurcations in a diffusive bimolecular model," Nonlinear Analysis: Real World Applications, vol. 11, no. 5, pp. 3770 3781, 2010.

[8] F. Yi, H. Zhang, A. Cherif, and W. Zhang, "Spatiotemporal patterns of a homogenous diffusive system modeling hair growth: Global asymptotical behavior and multiple bifurcation analysis," Communications on Pure and Applied Analysis, vol. 13, no. 1, pp. 347-369, 2014

[9] W. Han and Z. Bao, "Hopf bifurcation analysis of a reactiondiffusion Sel'kov system," Journal of Mathematical Analysis and Applications, vol. 356, no. 2, pp. 633-641, 2009.

[10] W. Ni and M. Tang, "Turing patterns in the Lengyel-Epstein system for the CIMA reaction," Transactions of the American Mathematical Society, vol. 357, no. 10, pp. 3953-3969, 2005. 


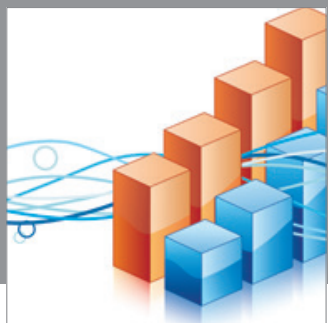

Advances in

Operations Research

mansans

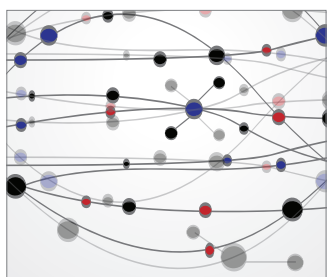

The Scientific World Journal
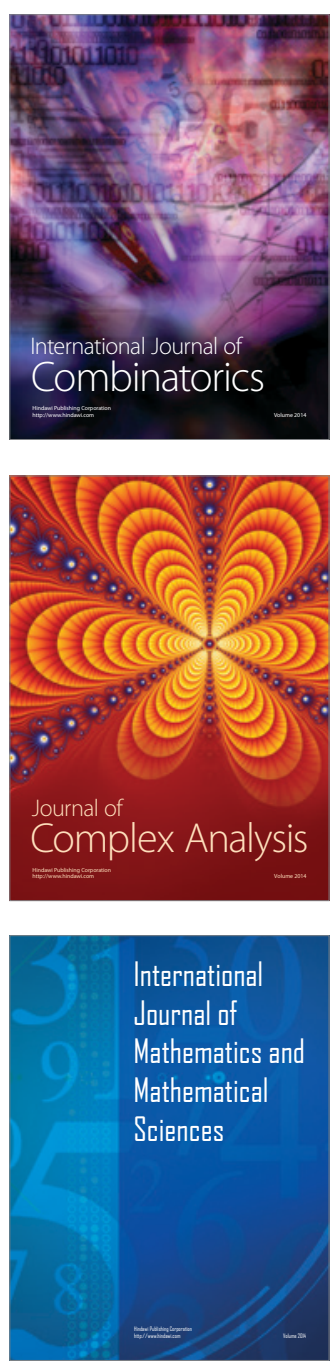
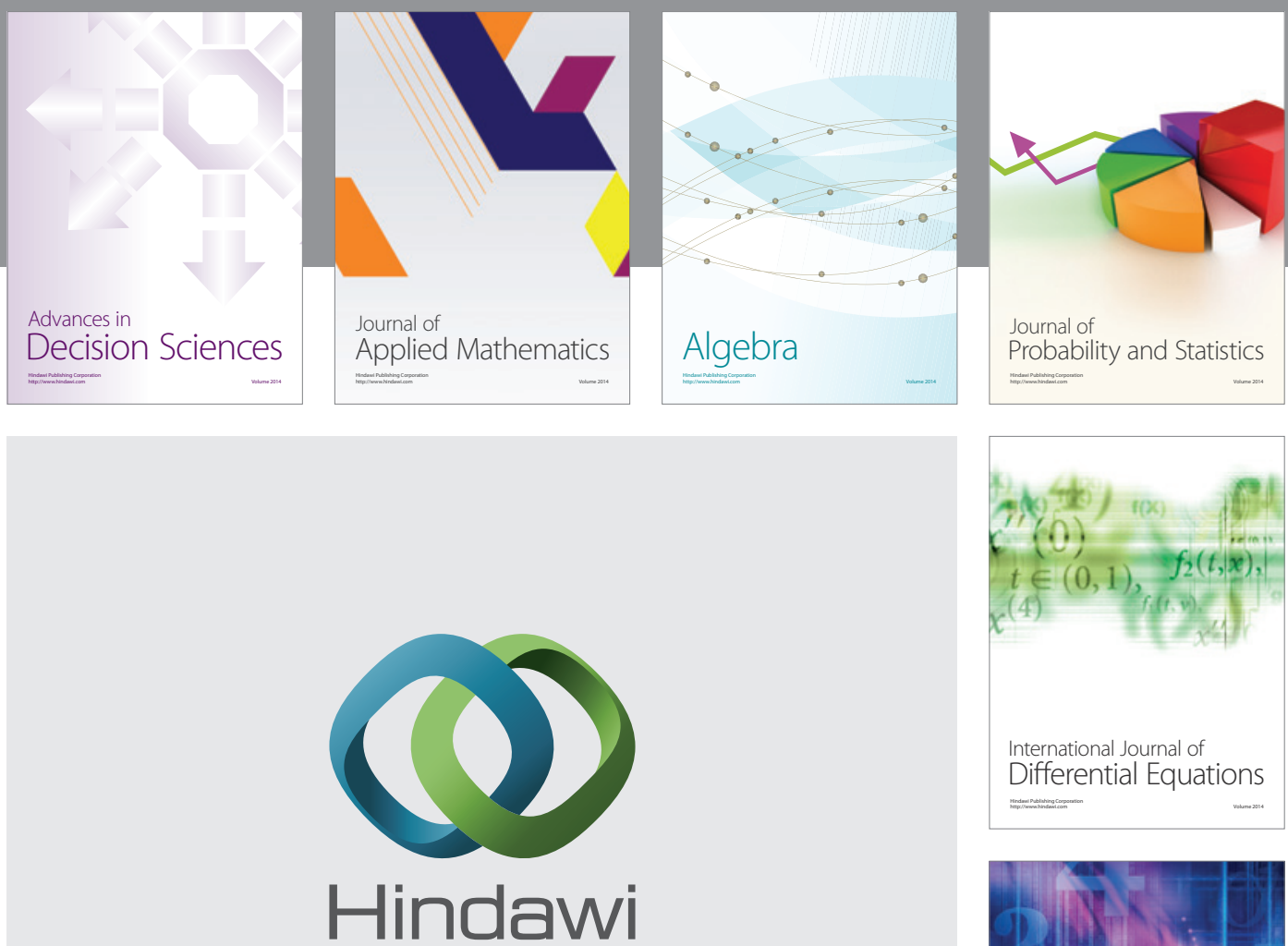

Submit your manuscripts at http://www.hindawi.com
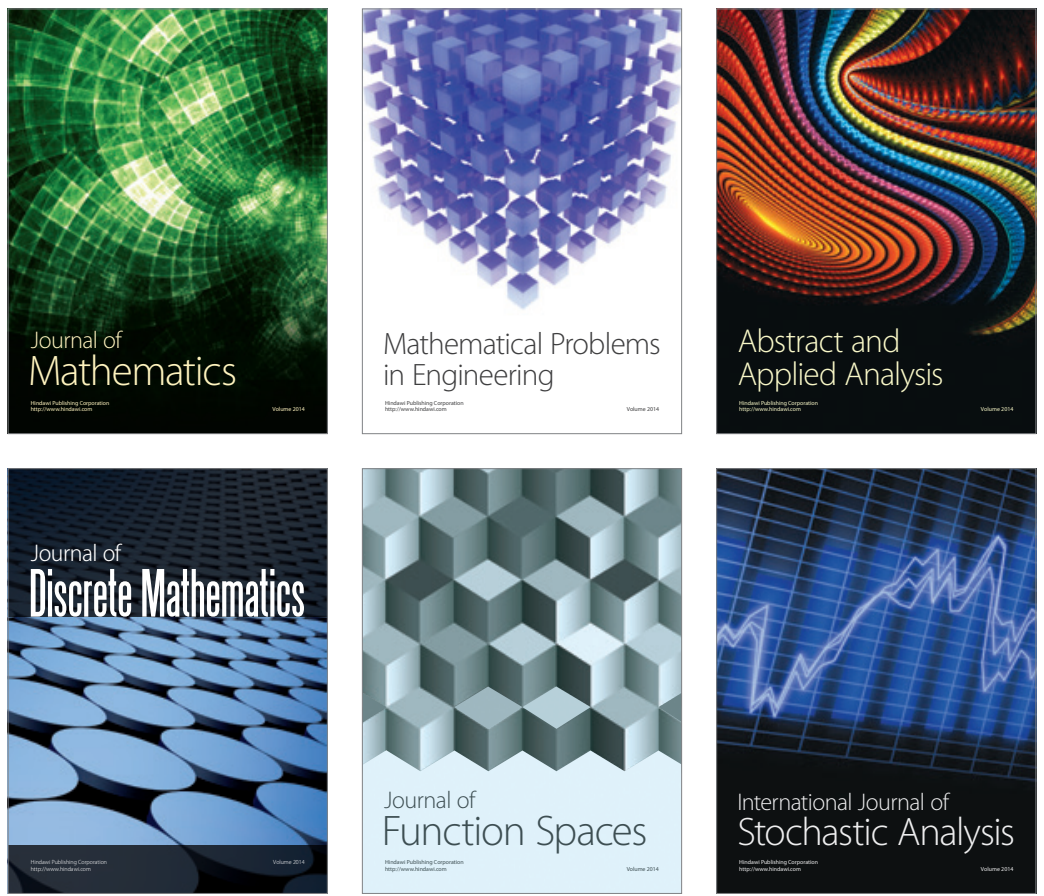

Journal of

Function Spaces

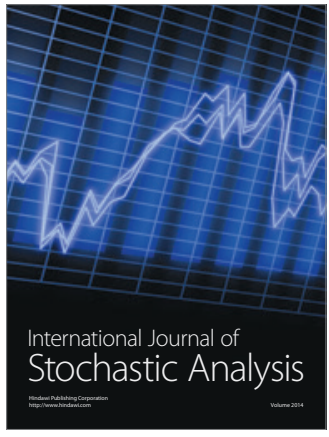

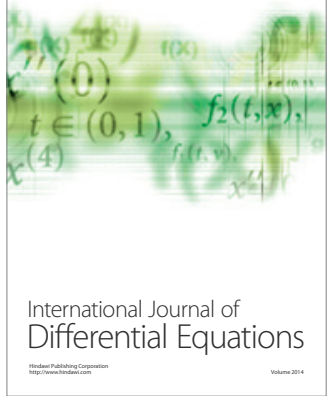
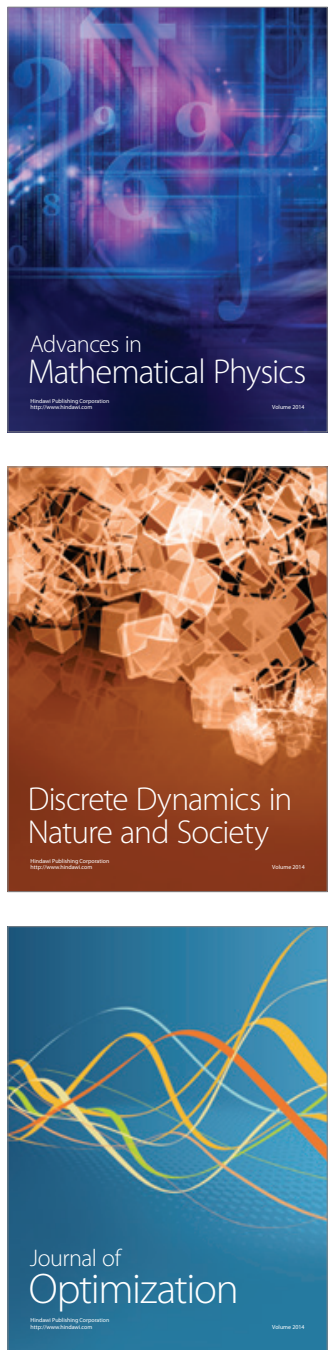